\title{
Radio spectral properties and the magnetic field of the SNR S147
}

\author{
L. Xiao ${ }^{1}$, E. Fürst ${ }^{2}$, W. Reich ${ }^{2}$, and J. L. $\operatorname{Han}^{1}$ \\ 1 National Astronomical Observatories, Chinese Academy of Sciences, Jia-20 DaTun Road, Chaoyang District, \\ Beijing 100012, PR China \\ e-mail: $[x l ; h j l] @ b a o . a c . c n$ \\ 2 Max-Planck-Institut für Radioastronomie, Auf dem Hügel 69, 53121 Bonn, Germany \\ e-mail: [efuerst;wreich] @mpifr-bonn.mpg.de
}

Received 10 August 2007 / Accepted 29 January 2008

\begin{abstract}
Context. S147 is a large faint shell-type supernova remnant (SNR) known for its remarkable spectral break at cm-wavelength, which is an important physical property to characterize SNR evolution. However, the spectral break is based on radio observations with limited precision.

Aims. New sensitive observations at high frequencies are required for a detailed study of the spectral properties and the magnetic field structure of S147.

Methods. We conducted new radio continuum and polarization observations of S147 at $\lambda 11 \mathrm{~cm}$ and at $\lambda 6 \mathrm{~cm}$ with the Effelsberg 100-m telescope and the Urumqi 25-m telescope, respectively. We combined these new data with published lower-frequency data from the Effelsberg 100-m telescope, and with very high-frequency data from WMAP to investigate the spectral turnover and polarization properties of $\mathrm{S} 147$.

Results. S147 consists of numerous filaments embedded in diffuse emission. We found that the integrated flux densities of S147 are $34.8 \pm 4.0 \mathrm{Jy}$ at $\lambda 11 \mathrm{~cm}$ and $15.4 \pm 3.0 \mathrm{Jy}$ at $\lambda 6 \mathrm{~cm}$. These new measurements confirm the known spectral turnover at $\sim 1.5 \mathrm{GHz}$, which can be entirely attributed to the diffuse emission component. The spectral index above the turnover is $\alpha=-1.35 \pm 0.20\left(\mathrm{~S} \sim v^{\alpha}\right)$. The filamentary emission component has a constant spectral index over the entire wavelength range up to $40.7 \mathrm{GHz}$ of $\alpha=-0.35 \pm 0.15$. The weak polarized emission of S147 is at the same level as the ambient diffuse Galactic polarization. The rotation measure of the eastern filamentary shell is about $-70 \mathrm{rad} \mathrm{m}^{-2}$.

Conclusions. The filamentary and diffuse emission components of S147 have different physical properties, which make S147 outstanding among shell type SNRs. We attribute the weak polarization of S147 at $\lambda 11 \mathrm{~cm}$ and at $\lambda 6 \mathrm{~cm}$ to a section of the S147 shell showing a tangetial magnetic field direction.
\end{abstract}

Key words. ISM: supernova remnants - radio continuum: ISM - techniques: polarimetric

\section{Introduction}

The extended object Shajn 147 (S147) has long, beautiful delicate filaments visible in the optical bands, and is located in the anti-center of the Galaxy. It was first classified as a possible shell-type supernova remnant (SNR) by Minkowski (1958) and van den Bergh (1960). The distance to S147 was estimated to be around $1 \mathrm{kpc}$ (Clark \& Caswell 1976; Kundu et al. 1980), using the so called $\Sigma$-D relation (Milne 1979). The interstellar reddening toward the SNR (Fesen et al. 1985) suggests a smaller distance of $0.8 \mathrm{kpc}$. This is supported by absorption lines of the B1e star HD 36665 at 880 pc originating from high velocity gas of S147 (Phillips et al. 1981; Sallmen \& Welsh 2004). The expansion velocity of the shell is about $80-120 \mathrm{~km} \mathrm{~s}^{-1}$ (Phillips et al. 1981; Kirshner \& Arnold 1979). The age of S147 was estimated to be $\sim 2 \times 10^{5} \mathrm{yr}$ through the radiative blast wave's Sedov-Taylor solution (Sofue et al. 1980; Kundu et al. 1980). The PSR J0538+2817 has been discovered within the boundary of S147 (Anderson et al. 1996), and is probably physically associated with the SNR (Anderson et al. 1996; Ng et al. 2007). The kinematic age of the pulsar, if originated from the SNR center, is

\footnotetext{
* Figures 1 and 2 are available in FITS format at the CDS via anonymous ftp to cdsarc.u-strasbg.fr $(130.79 .128 .5)$ or via http://cdsweb.u-strasbg.fr/cgi-bin/qcat?J/A+A/482/783
}

about $\sim 4 \times 10^{4} \mathrm{yr}$. The parallax of the pulsar suggests a distance of $1.47_{-0.27}^{+0.42} \mathrm{kpc}(\mathrm{Ng}$ et al. 2007).

DeNoyer (1974) summarized early radio maps of S147 between $178 \mathrm{MHz}$ and $1420 \mathrm{MHz}$. She obtained a flux density spectral index of $\alpha=-0.67 \pm 0.37$, revealing S147 as a SNR. Later, radio observations of $\mathrm{S} 147$ at $1648 \mathrm{MHz}$ and $2700 \mathrm{MHz}$ were made by Kundu et al. (1980), and the southern part of the SNR was observed at $4995 \mathrm{MHz}$ by Sofue et al. (1980). These high-frequency observations suggested a spectral turnover near $1.5 \mathrm{GHz}$ with a flat spectrum at lower frequencies and a steep spectrum at higher frequencies. The turnover needs to be confirmed due to large uncertainties in the low-frequency measurements and the fact that the observation at $4995 \mathrm{MHz}$ covered only the southern part of the remnant. Later sensitive measurements, at $1425 \mathrm{MHz}$ and $2695 \mathrm{MHz}$, by Fürst \& Reich (1986) could not improve the integrated radio spectrum, but revealed spectral index variations across S147. Regions of bright filaments show a flux density spectral index of about $\alpha=-0.5$, while regions in between show a spectral index near $\alpha=-1.0$. The observation at $863 \mathrm{MHz}$ by Reich et al. (2003) provided a more precise flux density at a low frequency, but did not improve on the uncertainty in the spectrum. The $2695 \mathrm{MHz}$ radio map made by Fürst \& Reich (1986) for the first time showed linearly polarized emission associated with some filamentary structure. 
Detailed information from this polarization observation cannot be derived due to an insufficient signal-to-noise ratio.

One goal of this work is to use the new sensitive $\lambda 6 \mathrm{~cm}$ receiver at the Urumqi $25-\mathrm{m}$ radio telescope to obtain a complete radio map at this band for a detailed study of the spectral turnover. Here we present results of total power and linear polarization maps at $4800 \mathrm{MHz}(\lambda 6 \mathrm{~cm})$. We also present new observations at $2639 \mathrm{MHz}(\lambda 11 \mathrm{~cm})$ with the Effelsberg $100-\mathrm{m}$ radio telescope and unpublished polarization data at $1420 \mathrm{MHz}(\lambda 21 \mathrm{~cm})$ obtained from the "Effelsberg Medium Galactic Latitude Survey" (Reich et al. 2004). The new observations and the data processing are described in Sect. 2 . The investigation of the integrated radio spectrum and possible spectral variations across the remnant are discussed in Sect. 4. The radio polarization and the rotation measure toward S147 are analyzed in Sect. 5. The results are summarized in Sect. 6.

\section{Observations and data processing}

New observations of S147 have been made with the Urumqi $25-\mathrm{m}$ radio telescope at $\lambda 6 \mathrm{~cm}$ and Effelsberg 100-m radio telescope at $\lambda 11 \mathrm{~cm}$. The main parameters are listed in Table 1.

\subsection{Observations at $\lambda 6 \mathrm{~cm}$}

We made continuum and polarization observations of S147 between January 2005 and February 2006. We scanned the large area of S147 along Galactic longitude or Galactic latitude direction. Five coverages of the field of $(\Delta l \times \Delta b)=\left(5^{\circ} \times 5^{\circ}\right)$ centered on $(l, b)=180^{\circ} .2,-1.7$ were observed in January 2005 , four coverages $(\Delta l \times \Delta b)=\left(4^{\circ} .2 \times 4^{\circ} .2\right)$ centered on $(l, b)=180^{\circ} .1,-1^{\circ} .5$ were added at the end of 2005, and five additional maps of $(\Delta l \times \Delta b)=\left(5^{\circ} \times 5^{\circ}\right)$ centered on $(l, b)=180^{\circ} .2,-1.6$ were observed again in February 2006. We moved the telescope with a scanning velocity between $2^{\prime} / \mathrm{s}$ and $3^{\prime} / \mathrm{s}$. The sampling and the pixel size of the map were $3^{\prime}$. There is one map scanned with a narrow bandwidth $(295 \mathrm{MHz}$ instead of $600 \mathrm{MHz}$ ) to mask interference from InSat (see Sun et al. 2007), so the effective total integration time is about $18.6 \mathrm{~s}$ per pixel and the theoretical sensitivity is about $0.2 \mathrm{mK} T_{\mathrm{A}}$, using $\sigma_{I}=T_{\text {sys }} / \sqrt{\Delta v \tau}$, where $T_{\text {sys }}$ is the system temperature, $T_{\mathrm{A}}$ is the antenna temperature, $\Delta v$ is the bandwidth, and $\tau$ is the integration time. The quoted sensitivity corresponds to $0.3 \mathrm{mK} T_{\mathrm{B}}$, where $T_{\mathrm{B}}$ is the brightness temperature.

The raw data from the four backend channels $\left(\mathrm{RR}^{*}, \mathrm{LL}^{*}\right.$, $\left.\mathrm{RL}^{*}, \mathrm{LR}^{*}\right)$, together with time information and telescope position were stored in the MBFITS format (Muders et al. 2007), and subsequently converted into the NOD2 format (Haslam 1974) to be further processed by the NOD2 based data reduction package under a Linux operation system. We edited the individual maps to remove any interference, to correct baseline curvatures by polynomial fitting and to suppress "scanning effects" by applying an "unsharp masking method" (Sofue \& Reich 1979) especially for the $U$ and $Q$ maps. Finally, we re-tabulated all maps into a map size of $5^{\circ} \times 5^{\circ}$ centered on $l, b=180^{\circ} .2,-1.7$ with a pixel size of 4 ', and "weaved" all maps together applying the method of Emerson \& Gräve (1988), which "destripes" a set of maps in the Fourier domain. We finally corrected the polarization map from the positive noise bias following Wardle \& Kronberg (1974).

The Urumqi $\lambda 6 \mathrm{~cm}$ total intensity map of S147 and polarization intensity map with vectors overlaid in magnetic field direction are shown in Fig. 1. The total intensity map clearly
Table 1. Observational parameters.

\begin{tabular}{lll}
\hline \hline Wavelength & $\lambda 6 \mathrm{~cm}$ & $\lambda 11 \mathrm{~cm}$ \\
Frequency & $4800 \mathrm{MHz}$ & $2639 \mathrm{MHz}$ \\
Bandwidth & $600 \mathrm{MHz}$ & $80 \mathrm{MHz}$ \\
$H P B W[']$ & 9.5 & 4.4 \\
aperture efficiency[\%] & 62 & 53 \\
beam efficiency[\%] & 67 & 58 \\
$T_{\text {sys }}[\mathrm{K}]$ & 22 & 17 \\
$T_{\mathrm{B}}[\mathrm{K}] / \mathrm{S}[\mathrm{Jy}]$ & 0.164 & 2.60 \\
Main Calibrator & $3 \mathrm{C} 286$ & $3 \mathrm{C} 286$ \\
Flux Density & $7.5 \mathrm{Jy}$ & $11.5 \mathrm{Jy}$ \\
Polarization Percentage & $11.3 \%$ & $9.9 \%$ \\
Polarization Angle & $33^{\circ}$ & $33^{\circ}$ \\
No. of coverages & 14 & 6 \\
pixel integration time [s] & 18.6 & 3 \\
rms (total intensity) [mK] & $0.7^{*}$ & 5.0 \\
rms (polarized intensity) [mK] & $0.3^{*}$ & 3.5 \\
\hline
\end{tabular}

${ }^{*}$ The quoted sensitivity at $\lambda 6 \mathrm{~cm}$ is valid for the area $l=178^{\circ} 0$ to $l=$ $182^{\circ} .2, b=-3.6$ to $b=-0.6$ (see text).

reveals the spherical SNR shell with a hollow region in its center. The rms-noise in the common area of all coverages (see Table 1) in total intensity is $0.7 \mathrm{mK} T_{\mathrm{B}}$ and in polarized intensity is $0.3 \mathrm{mK} T_{\mathrm{B}}$. These values are slightly larger than expected. It could be partially caused by confusion. Uyanıker et al. (1999) found a confusion limit of $15 \mathrm{mK} T_{\mathrm{B}}$ at $1400 \mathrm{MHz}$, which converts to $0.48 \mathrm{mK} T_{\mathrm{B}}$ at $\lambda 6 \mathrm{~cm}$ based on a flux density spectral index of $\alpha=-0.9$ (Zhang et al. 2003). Combining this value with the theoretically expected value, we obtain an rms-noise of $0.5 \mathrm{mK} T_{\mathrm{B}}$. Another part may be explained by residual gain fluctuations, "scanning effects", atmospheric and ground radiation variations, and low-level interference close to the noise level (Sun et al. 2006).

\subsection{Observations at $\lambda 11 \mathrm{~cm}$}

We observed $\mathrm{S} 147$ at $\lambda 11 \mathrm{~cm}$ with a new receiver installed in 2005 in the secondary focus of the Effelsberg $100-\mathrm{m}$ radio telescope. The map size is $5^{\circ} \times 5^{\circ}$ centered at $l, b=180^{\circ} .2,-1^{\circ} .6$. We made six coverages along Galactic longitude and Galactic latitude direction with a scanning velocity of $4^{\prime} / \mathrm{s}$. We processed the maps in the NOD2 format based standard reduction package for observations with the Effelsberg 100-m telescope. Bad data caused by strong interference were blanked. Scanning effects were suppressed by using the method of "unsharp masking" developed by Sofue \& Reich (1979). Finally, we "weaved" together all edited maps applying the method of Emerson \& Gräve (1988).

The $\lambda 11 \mathrm{~cm}$ maps are shown in Fig. 2. The rms-noise in total intensity (upper panel) is measured to be $5.0 \mathrm{mK} T_{\mathrm{B}}$. In polarized intensity, we found a value of $3.5 \mathrm{mK} T_{\mathrm{B}}$. Because of the higher angular resolution of 4.4 , the shell and the filamentary structures can be more clearly identified than in the $\lambda 6 \mathrm{~cm}$ map.

\section{Comparison of radio and optical observations}

We used the $\lambda 11 \mathrm{~cm}$ total intensity map at the original angular resolution after subtraction of the point-like sources listed in Table 2 to separate small-scale and large-scale structures applying the "background filtering" (BGF) procedure, invented by Sofue \& Reich (1979), where a filtering beam of $12^{\prime}$ was used. A comparison of the small-scale radio structure with optical filaments of S147 is displayed in Fig. 3, which demonstrates an 

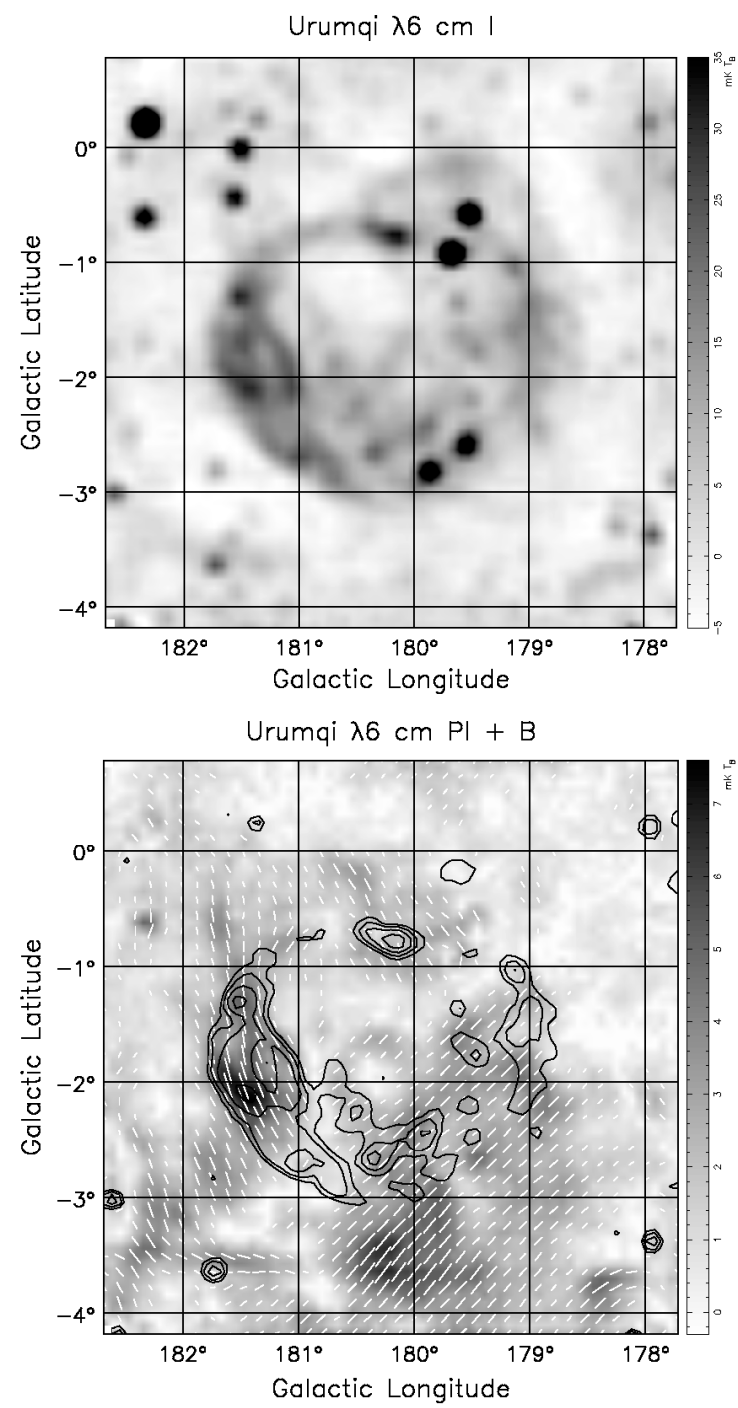

Fig. 1. The Urumqi $\lambda 6 \mathrm{~cm}$ map of S147 at an angular resolution of 9.5. The upper panel shows the total intensity map. The lower panel shows the polarization intensity map with contours of total intensities overlaid (the strong point-like sources listed in Table 2 have been subtracted). Contours start at $10 \mathrm{mK} T_{\mathrm{B}}$, and increase by a factor of $\sqrt{2}$. The bars show the orientation of the magnetic field $\left(\mathrm{E}+90^{\circ}\right)$ for the case of negligible Faraday rotation. Their length is proportional to the polarized intensity.

excellent agreement, even faint optical structures of the blowouts toward north-east and south-west are visible in both bands.

We also overlay the radio filamentary structures on the $\mathrm{H}_{\alpha}$ map compiled by Finkbeiner (2003) in Fig. 4, and find a rather good positional coincidence when inspecting individual filaments. However, we find large variations in the relative intensities, which have been already mentioned by Sofue et al. (1980). These variations may be explained by either stronger magnetic fields in the radio bright filaments and/or lower temperatures/densities in the optically-bright filaments. The measured temperature and density variations (Fesen et al. 1985; D'Odorico \& Sabbadin 1977) support this conclusion. D'Odorico \& Sabbadin (1977) obtained an average electron density of about $250 \mathrm{~cm}^{-3}$ assuming an electron temperature of $10^{4} \mathrm{~K}$ and showed that the compression by an adiabatic shock is insufficient to obtain these densities. Obviously, at least parts of S147 are already in the cooling phase of SNR evolution.
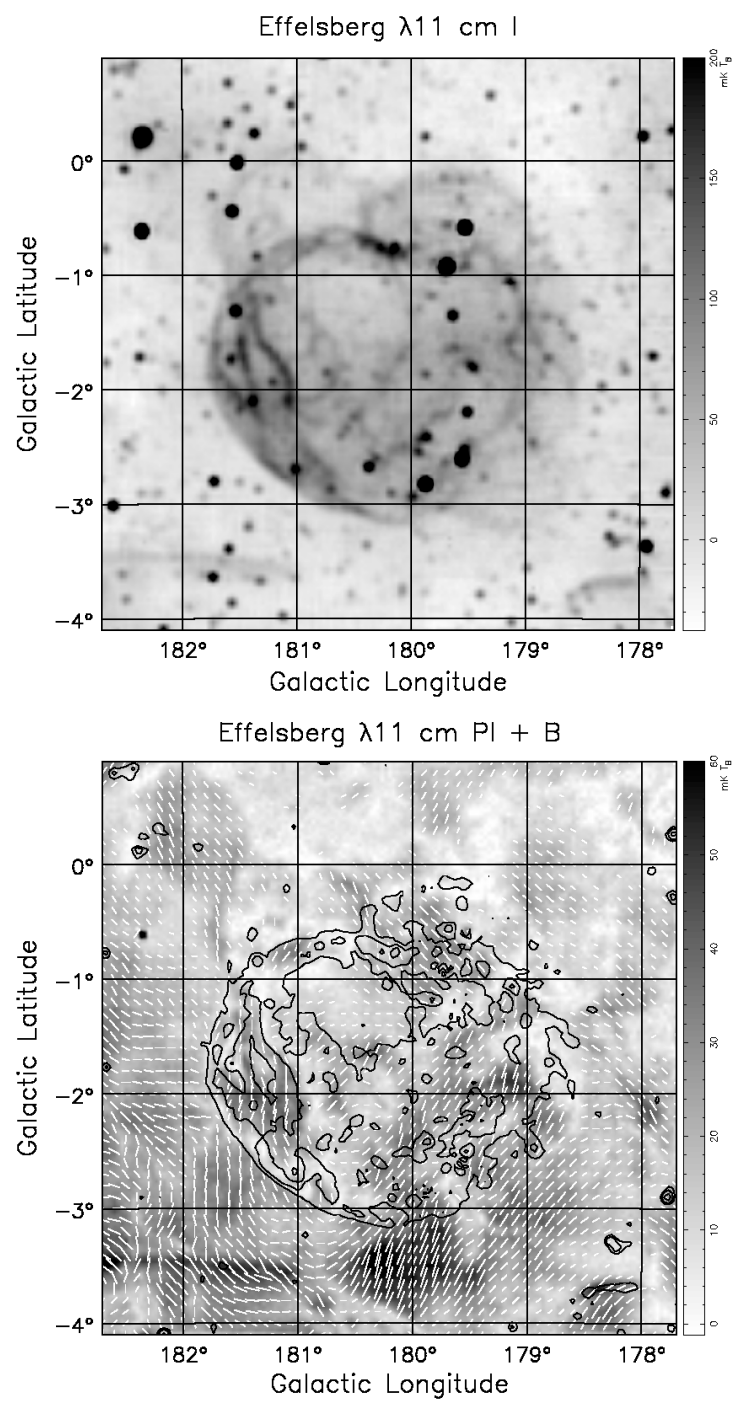

Fig. 2. Same as Fig. 1 but showing the Effelsberg $\lambda 11 \mathrm{~cm}$ maps at an angular resolution of 4!.4. Contours start from $50 \mathrm{mK} \mathrm{T}$ and increase by a factor of 2 .

\section{Spectral properties of $\mathrm{S} 147$}

\subsection{The integrated radio spectrum of $S 147$}

The new observations with the Urumqi $25-\mathrm{m}$ radio telescope provide the first complete map of $\mathrm{S} 147$ at $\lambda 6 \mathrm{~cm}$. In order to estimate the integrated flux density of S147, we subtracted eleven strong point-like sources (see Table 2) toward S147 from the $\lambda 6 \mathrm{~cm}$ and $\lambda 11 \mathrm{~cm}$ maps. The flux densities of these sources have previously been listed by Fürst et al. (1982), and agree within 0.1 Jy to those in Table 2. We calculated the integrated flux density with the method of ring integration by summing up the emission in concentric rings centered on $l, b=180^{\circ} .2,-1^{\circ} .6$. The integration stopped at a radius of 2.0 . Beyond this radius, we found an average base-level of $8.7 \mathrm{mK} T_{\mathrm{B}}$ at $\lambda 11 \mathrm{~cm}$ and of $1.9 \mathrm{mK} T_{\mathrm{B}}$ at $\lambda 6 \mathrm{~cm}$. We have subtracted these base-levels from the maps before calculating the flux density of S147. Taking these local base-levels into account, polygon integrations at the two wavelengths just outside the periphery of S147 result in the same flux density values as obtained from the method of ring integration. From variations just outside S147 we found the uncertainty in the base-levels to be $2 \mathrm{mK} T_{\mathrm{B}}$ at $\lambda 11 \mathrm{~cm}$ and $1 \mathrm{mK} T_{\mathrm{B}}$ at $\lambda 6 \mathrm{~cm}$. Considering $5 \%$ of other uncertainties including calibration, the 
Table 2. Strong point-like sources in the boundary of $S 147$ detected by Effelsberg at $\lambda 11 \mathrm{~cm}$ and Urumqi at $\lambda 6 \mathrm{~cm}$. The flux density is given by the peak flux of two-dimensional Gaussian fits.

\begin{tabular}{cccccrr}
\hline \hline Source & $\begin{array}{c}\alpha_{1950} \\
(\mathrm{~h} \mathrm{~m} \mathrm{~s})\end{array}$ & $\begin{array}{c}\delta_{1950} \\
\left({ }^{\circ}{ }^{\prime \prime}\right)\end{array}$ & $\begin{array}{c}\alpha_{2000} \\
(\mathrm{~h} \mathrm{~m} \mathrm{~s})\end{array}$ & $\begin{array}{c}\delta_{2000} \\
\left({ }^{\circ}{ }^{\prime \prime}\right)\end{array}$ & $\begin{array}{r}S_{11 \mathrm{~cm}} \\
(\mathrm{mJy})\end{array}$ & $\begin{array}{r}S_{6 \mathrm{~cm}} \\
(\mathrm{mJy})\end{array}$ \\
\hline $0531+275$ & 053118.3 & 273024 & 053426.6 & 273223 & $533 \pm 37$ & $287 \pm 44$ \\
$0531+279$ & 053121.7 & 275341 & 053430.6 & 275539 & $383 \pm 33$ & $265 \pm 24$ \\
$0533+282$ & 053248.2 & 280924 & 053557.5 & 281116 & $135 \pm 11$ & $51 \pm 14$ \\
$0533+272$ & 053305.6 & 271043 & 053613.5 & 271233 & $115 \pm 13$ & $84 \pm 13$ \\
$0534+284$ & 053413.5 & 282441 & 053723.2 & 282626 & $107 \pm 13$ & $130 \pm 20$ \\
$0535+266$ & 053433.1 & 263725 & 053740.2 & 263909 & $86 \pm 12$ & \\
$0536+285$ & 053620.5 & 283019 & 053930.4 & 283156 & $133 \pm 10$ & $71 \pm 18$ \\
$0538+287$ & 053806.3 & 284114 & 054116.5 & 284243 & $762 \pm 46$ & $527 \pm 47$ \\
$0539+290$ & 053902.7 & 290020 & 054213.4 & 290145 & $665 \pm 42$ & $435 \pm 33$ \\
$0541+269$ & 054103.4 & 265507 & 054411.0 & 265623 & $146 \pm 16$ & \\
$0544+273$ & 054427.5 & 272058 & 054735.7 & 272159 & $262 \pm 17$ & $251 \pm 22$ \\
\hline
\end{tabular}
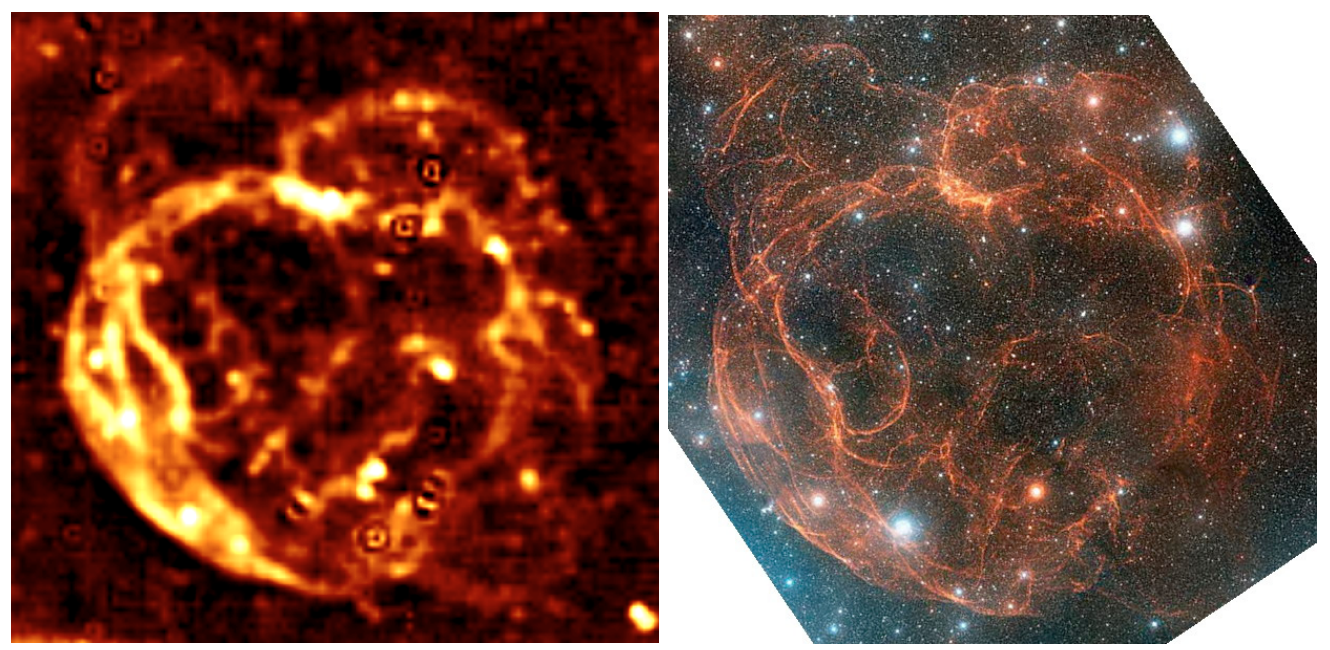

Fig. 3. Left panel: the filamentary structure of $\mathrm{S} 147$ at $\lambda 11 \mathrm{~cm}$ with most of strong point-like sources and diffuse large-scale $\left(>12^{\prime}\right)$ emission component subtracted (see text for details). Right panel: the optical image of S147 (Credit: Digitized Sky Survey, ESA/ESO/NASA, http:// antwrp.gsfc. nasa.gov/apod/ap051129.html).

flux density values are $34.8 \pm 4.0 \mathrm{Jy}$ at $\lambda 11 \mathrm{~cm}$ and $15.4 \pm 3.0 \mathrm{Jy}$ at $\lambda 6 \mathrm{~cm}$. Figure 5 displays the integrated spectrum of S147 including available low-frequency flux density data.

Reich et al. (2003) placed the turnover frequency between $1.5 \mathrm{GHz}$ and $2 \mathrm{GHz}$ using the extrapolated flux density of the incomplete $5 \mathrm{GHz}$ map observed by Sofue et al. (1980). The extrapolated flux density value as well as the spectral turnover at $\sim 1.5 \mathrm{GHz}$ are clearly confirmed by new measurements at $\lambda 6 \mathrm{~cm}$ and $\lambda 11 \mathrm{~cm}$. The spectrum is flat at lower frequencies and steepens toward frequencies higher than $1 \mathrm{GHz}$ to $2 \mathrm{GHz}$. The low-frequency spectrum has a spectral index of about $\alpha \sim$ $-0.30 \pm 0.15$. The spectral index is about $\alpha \sim-1.20 \pm 0.30$ above the break frequency. The relatively large error at high frequencies is mainly caused by the still large uncertainty of the flux density at $4800 \mathrm{MHz}(\lambda 6 \mathrm{~cm})$, which, in principle, is very difficult to reduce for faint large diameter objects like S147 located in the Galactic plane.

\subsection{The spectral index map of $S 147$}

The Urumqi $\lambda 6 \mathrm{~cm}$ and the Effelsberg $\lambda 11 \mathrm{~cm}$ maps with pointlike sources and constant base-levels removed were both convolved to a common angular resolution of $10^{\prime}$. We estimated the relative position accuracy of the two maps by using the positions of point-like sources from Table 2. We found the mean positional difference to be 6 " with a maximum of $28^{\prime \prime}$.

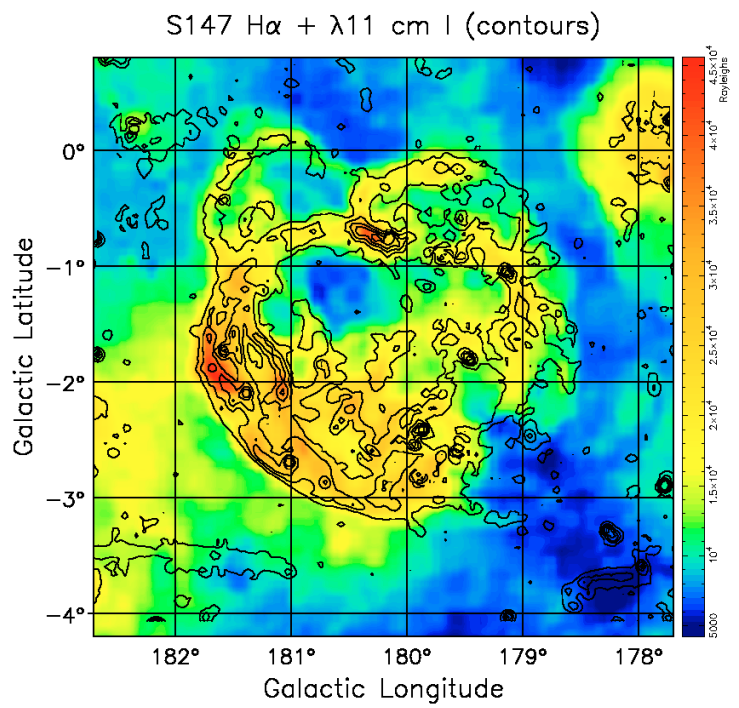

Fig. 4. The filamentary structures of $\mathrm{S} 147$ observed at $\lambda 11 \mathrm{~cm}$ is overlaid with $\mathrm{H}_{\alpha}$ map (Finkbeiner 2003). The angular resolution of the $\mathrm{H}_{\alpha}$ map is $6^{\prime}$. The contours at $\lambda 11 \mathrm{~cm}$ start at $20 \mathrm{mK} T_{\mathrm{B}}$ and run in steps of $35 \mathrm{mK} T_{\mathrm{B}}$.

We display the spectral index map between $\lambda 11 \mathrm{~cm}$ and $\lambda 6 \mathrm{~cm}$ in Fig. 6. We calculated the spectral index of each pixel 


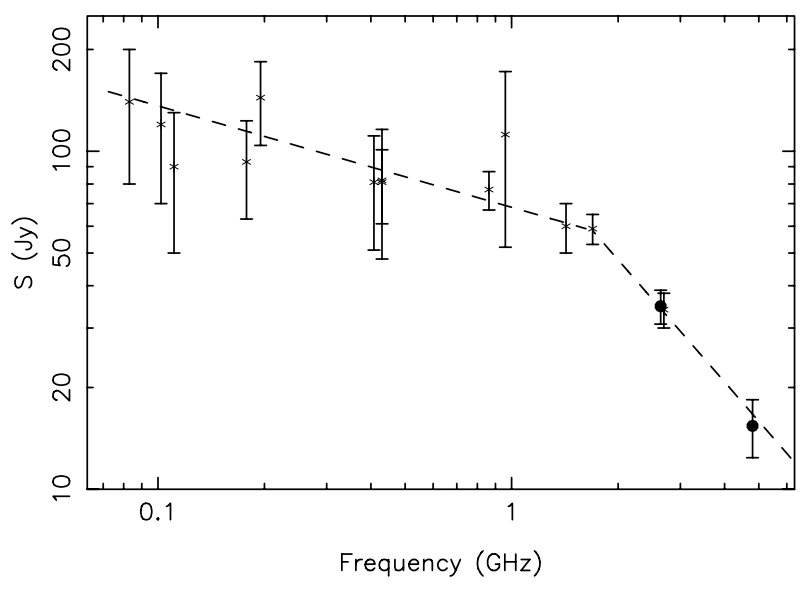

Fig. 5. Spectrum of the integrated radio flux densities of S147. The lowfrequency spectrum has a spectral index of $\alpha \sim-0.30 \pm 0.15$. Above the break the spectral index is $\alpha \sim-1.20 \pm 0.30$. The S147 integrated flux densities are taken from Fürst \& Reich (1986); Reich et al. (2003); and Kovalenko et al. (1994). We omitted the $\lambda 6 \mathrm{~cm}$ flux density value obtained by Sofue et al. (1980), because this measurement did not cover the entire source. Reich et al. (2003) have corrected the values for the contribution of point-like sources as listed by Fürst et al. (1982), assuming a mean source spectral index of $\alpha=-0.75$ at frequencies lower than $408 \mathrm{MHz}$.

from the brightness temperatures at the two frequencies. In order to achieve accurate spectral indices and to exclude the influence of systematic effects we set a lower flux density limit of $40 \mathrm{mK} T_{\mathrm{B}}$ and $6 \mathrm{mK} T_{\mathrm{B}}$ for the $\lambda 11 \mathrm{~cm}$ and $\lambda 6 \mathrm{~cm}$ map, respectively. The possible variations of the base-levels at $\lambda 11 \mathrm{~cm}$ and $\lambda 6 \mathrm{~cm}$ cause an uncertainty of the spectral indices of $\Delta \alpha \sim 0.3$. The uncertainty is largest where the total intensity is small. The general result of a much flatter radio spectrum of the filaments compared with the large-scale component is not altered. However, systematic errors make it difficult to derive positional spectral index variations of the large scale component. The spectrum for regions with bright radio filaments, particularly in the eastern and northern part of S147, is much flatter than for regions with less pronounced filaments and for the large-scale diffuse emission component near the center. As seen in the total intensity maps, faint, extended Galactic emission exists outside of S147. Such emission features may also exist in the projected region toward S147, leading to variations of the spectral index.

\subsection{T-T plot analysis}

The temperature-temperature $(T-T)$ plot is a method (Turtle et al. 1962) to investigate the spectral behavior, independent from a constant base-level across the source. We used the maps at an angular resolution of $10^{\prime}$ at $\lambda 11 \mathrm{~cm}$ and $\lambda 6 \mathrm{~cm}$ to check the spectral index of the filamentary and diffuse emission components. We first decomposed both maps into small-scale filamentary structures and large-scale diffuse emission component with the BGF procedure described by Sofue \& Reich (1979) with a filtering beam of $18^{\prime}$ and cut-values of $100 \mathrm{mK} T_{\mathrm{B}}$ at $\lambda 11 \mathrm{~cm}$ and $25 \mathrm{mK} T_{\mathrm{B}}$ at $\lambda 6 \mathrm{~cm}$. The resultant $T T$-plots for the filaments and for the diffuse emission component are shown in Fig. 7. They have largely different spectral indices. We found the steep spectrum with a spectral index of $\alpha=-1.35 \pm 0.20$ for the diffuse emission component, while we found a spectral index $\alpha=-0.43 \pm 0.10$ for the filamentary emission component. This index is slightly steeper than the integrated low-frequency

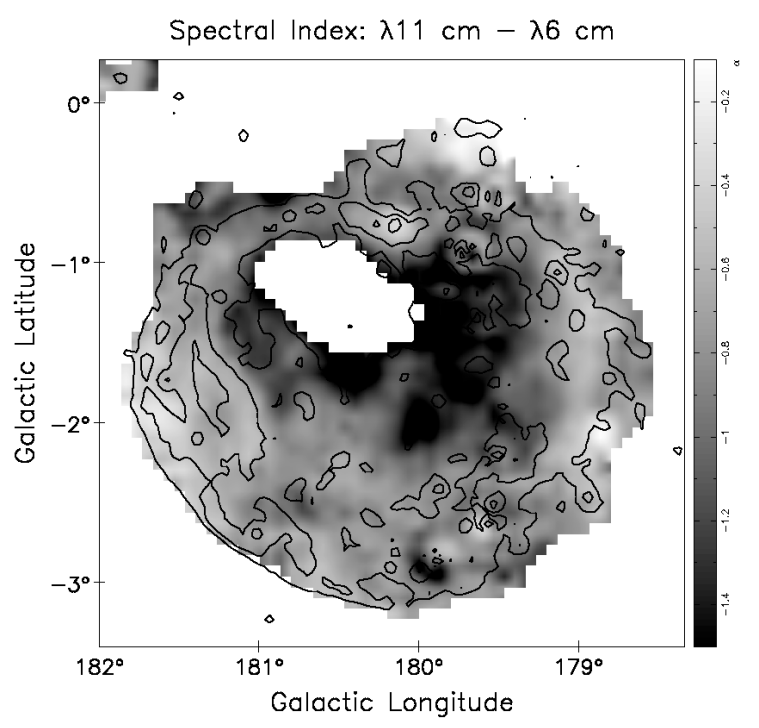

Fig. 6. Spectral index map calculated between $\lambda 11 \mathrm{~cm}$ and $\lambda 6 \mathrm{~cm}$ at $10^{\prime}$ angular resolution. The overlaid contours represent total intensities at $\lambda 11 \mathrm{~cm}$ (with point-like sources listed in Table 2 subtracted), starting from $50 \mathrm{mK} T_{\mathrm{B}}$ and increasing by a factor of 2 .

spectrum ( $\alpha=-0.30 \pm 0.15$ ), but is comparable considering the quoted uncertainties. The quoted errors include the statistical error (number of data points reduced to one per $H P B W$ ) as well as the error, which follows from fitting the data twice, alternatively taking the data of one of the two wavelengths as independent variable. The result in Fig. 7 may be affected by possible variations of the base-levels across S147. This was inspected using $T T$-plots of four smaller regions separated by $l=180^{\circ} .0$ and $b=-1.9$. For the filamentary component, the variation of the spectral index is within the quoted error of $\Delta \alpha= \pm 0.10$. Therefore, the filamentary emission component probably has a fairly straight radio spectrum over the accessible frequency range. For the diffuse emission component the spectral index varies between $\alpha=-1.10$ and $\alpha=-1.56$. There is no doubt that the radio spectrum of the diffuse emission component is much steeper than that of the filamentary component. This is in agreement with the result from the spectral index map. It is entirely the diffuse emission component that produces the spectral turnover around $1.5 \mathrm{GHz}$. The integrated flux densities of the separated diffuse and filamentary emission components are $\sim 22.8 \mathrm{Jy}$ and $\sim 12 \mathrm{Jy}$ at $\lambda 11 \mathrm{~cm}$, and $\sim 8.4 \mathrm{Jy}$ and $\sim 7 \mathrm{Jy}$ at $\lambda 6 \mathrm{~cm}$, respectively, which also shows the difference of spectral indices of the two components.

It is clear that above $4800 \mathrm{MHz}$ the flux density of the radio filaments dominates the integrated flux density of S147, causing a spectral flattening toward higher frequencies. To check this, we inspected the WMAP data in all five bands between $22.8 \mathrm{GHz}$ and $93.5 \mathrm{GHz}$ (Hinshaw et al. 2007) for a signature of S147. We are able to clearly trace S147 up to the highest frequency especially its region with strong radio filaments near $l=181^{\circ}$.

We convolved all WMAP data and the $\lambda 11 \mathrm{~cm}$ map to the beam size of the $22.8 \mathrm{GHz}$ map $\left(49^{\prime}\right)$. We separated the filamentary emission component of S147 in all maps by applying the BGF procedure described by Sofue \& Reich (1979), using a filtering beam size of $50^{\prime}$. As an example, we show the WMAP data at $40.7 \mathrm{GHz}$ in Fig. 8 at $49^{\prime}$ angular resolution in comparison with the filamentary emission component of the Effelsberg $\lambda 11 \mathrm{~cm}$ map at the original angular resolution. To check the spectral index, we performed a $T-T$ Plot (Fig. 9) for the 


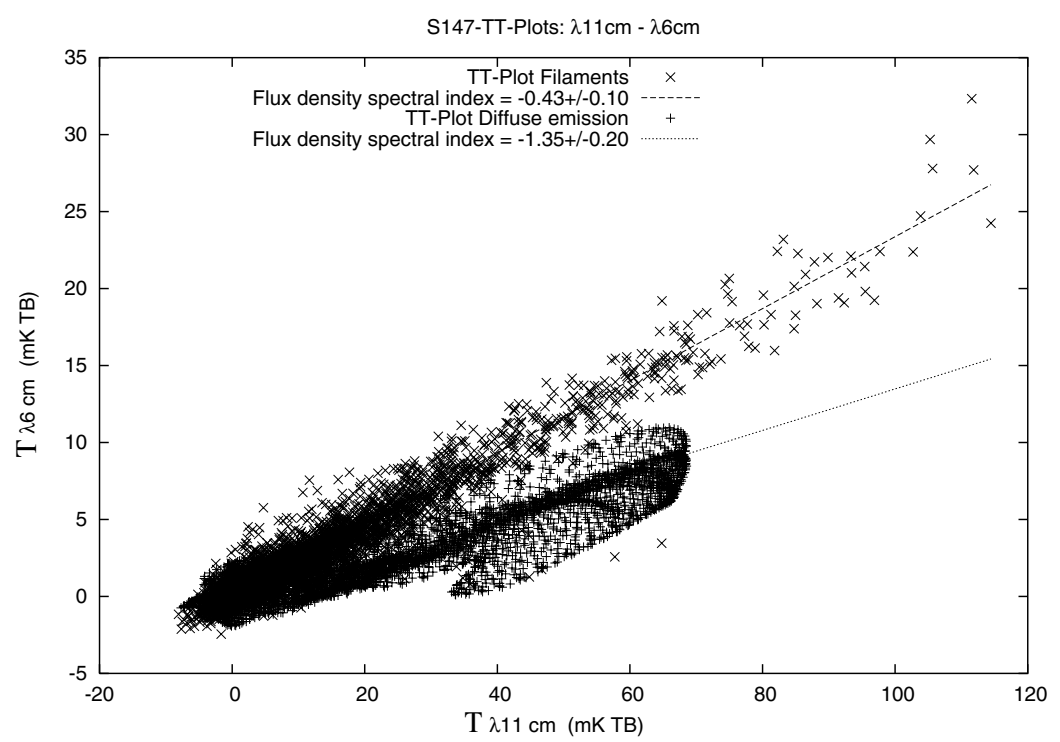

Fig. 7. The $T-T$ plot for the filamentary and diffuse emission components of $\mathrm{S} 147$ between $\lambda 11 \mathrm{~cm}$ and $\lambda 6 \mathrm{~cm}$. Filamentary emission component in general has a spectral index of $\alpha=-0.43 \pm 0.10$. The diffuse emission component has a spectral index of $\alpha=-1.35 \pm 0.20$.

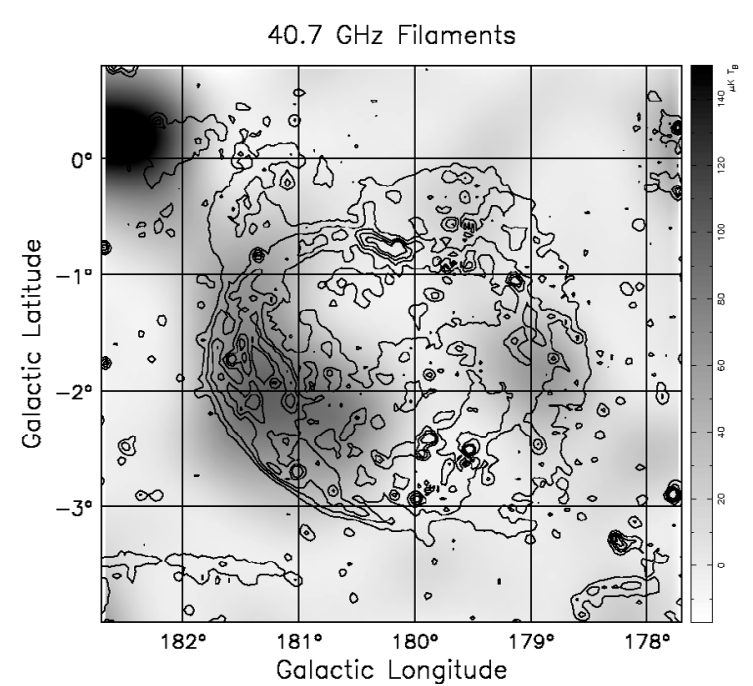

Fig. 8. The filamentary emission component of $\mathrm{S} 147$ at $40.7 \mathrm{GHz}$ (grayscale) observed by WMAP at an angular resolution of $49^{\prime} . \lambda 11 \mathrm{~cm}$ contours of Effelsberg measurements at an angular resolution of 4.4 start at $20 \mathrm{mK} T_{\mathrm{B}}$ and run in steps of $35 \mathrm{mK} T_{\mathrm{B}}$.

filamentary emission component at $40.7 \mathrm{GHz}$ and $\lambda 11 \mathrm{~cm}$ of the area $l=180.8$ to $l=181^{\circ} .9, b=-0.87$ to $b=-2.7$. We found the spectral index to be $\alpha=-0.31 \pm 0.20$, which is consistent with that between $\lambda 11 \mathrm{~cm}$ and $\lambda 6 \mathrm{~cm}$. A similar spectral index for the filamentary emission component is obtained between $\lambda 11 \mathrm{~cm}$ and $22.8 \mathrm{GHz}$ and $33.0 \mathrm{GHz}$, respectively.

From the various methods we may derive a spectral index for the filamentary emission component of $\alpha=-0.35 \pm 0.15$. Using the flux density of $7 \mathrm{Jy}$ for the filamentary structures at $\lambda 6 \mathrm{~cm}$, we calculate the flux density of filamentary emission component to $3.5 \mathrm{Jy}$ at $40.7 \mathrm{GHz}, 4.3 \mathrm{Jy}$ at $22.8 \mathrm{GHz}$, and $3.8 \mathrm{Jy}$ at $33.0 \mathrm{GHz}$. The flux density of the diffuse emission component of $\mathrm{S} 147$ at $40.7 \mathrm{GHz}$ or other WMAP frequencies can not be determined since a separation of this component from the underlying stronger large-scale Galactic emission is not possible.

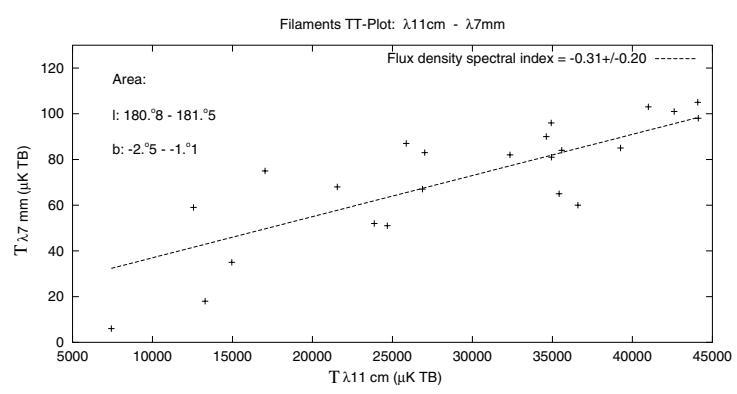

Fig. 9. The $T-T$ Plot of filamentary emission component at $\lambda 7 \mathrm{~mm}$ $(40.7 \mathrm{GHz}$ ) and $\lambda 11 \mathrm{~cm}$. The spectral index is $\alpha=-0.31 \pm 0.20$.

At $60.8 \mathrm{GHz}$ and $93.5 \mathrm{GHz}$ the emission in the quoted area increases significantly, which is most likely due to thermal dust emission associated with the SNR.

We show all flux densities up to $40.7 \mathrm{GHz}$ in Fig. 10, where the WMAP values miss the small contribution from the diffuse emission component. In addition, we have plotted the supposed straight spectrum of the filamentary component with a spectral index of $\alpha=-0.35$. We have also plotted the contribution of the diffuse large-scale emission component and the sum of both. The turnover of the integrated total intensity spectrum has to be attributed to the turnover of the large-scale emission component. We assume that both emission components have the same spectral index below the break frequency. In summary, the integrated total intensity spectrum is flat at low frequencies, steep between $1 \mathrm{GHz}$ and about $10 \mathrm{GHz}$, and flattens again toward higher frequencies. No other SNR is known showing a similar radio spectrum. Hence, the radio spectrum of S147 is unique.

\subsection{The analysis of the spectral turnover}

A spectral turnover is very rare for shell-type SNRs. Some early claims have turned out to result from limited accuracy of highfrequency measurements. S147 is the best known case for a spectral break so far.

Several possible mechanisms cause a spectral turnover: (1) the presence of two populations with different energy spectra; (2) shock acceleration of particles; (3) the compression of 


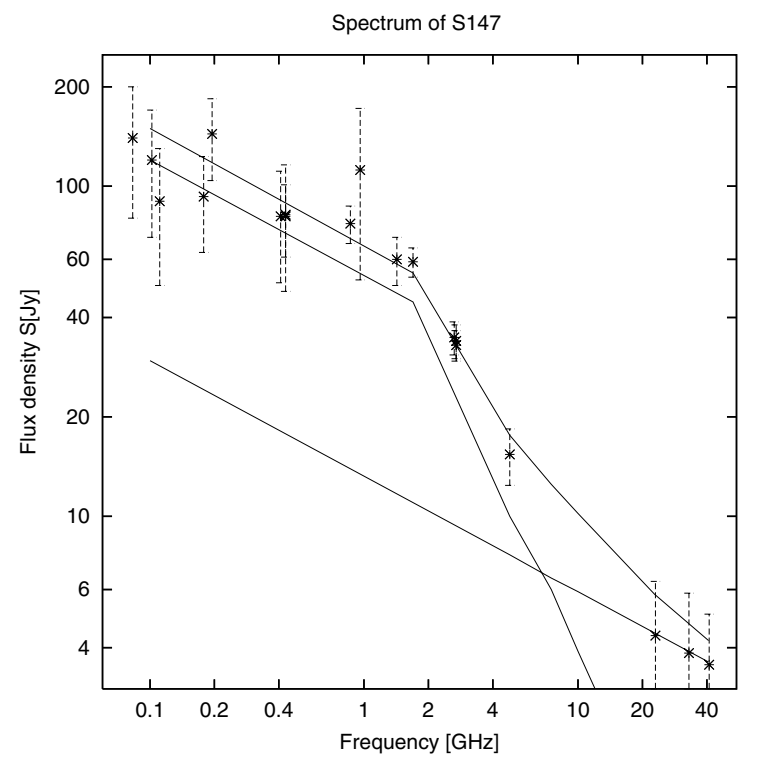

Fig. 10. Integrated total intensity spectrum of S147, including WMAP data for the filaments. The weaker contribution from the diffuse emission component can not be obtained from the WMAP data. The model spectrum of the filamentary component, the diffuse component with a spectral break at $1.7 \mathrm{GHz}$, and the sum of both are shown in addition (see text).

the Galactic magnetic field; and (4) synchrotron losses (aging) of electrons.

(1), (2) According to Leahy \& Roger (1998) the first two mechanisms may not be relevant for a synchrotron spectrum as given in Fig. 5.

(3) The Galactic relativistic electron spectrum has a slow turnover due to various loss and propagation effects over time. Webster (1974) has compiled the Galactic radio spectrum, which varies from $\alpha \sim-0.5$ to $\alpha \sim-0.8$ between $\sim 100 \mathrm{MHz}$ and $\sim 600 \mathrm{MHz}$. The corresponding electron energy index varies from $\sim 2.0$ to $\sim 2.6$ over the energy range $2.6 \mathrm{GeV}$ to $6.5 \mathrm{GeV}$ in case the magnetic field strength is $3 \mu \mathrm{G}$. Direct observations of the electron spectrum (Tang 1984) also reveal a change of the Galactic electron spectral index from 2.0 at low energy $(<2 \mathrm{GeV})$ to 3.5 at higher energies. Due to the shock wave of the SNR, the compressed local magnetic field in a SNR shifts the spectral turnover toward a higher frequency, e.g., that at about $1.5 \mathrm{GHz}$ of S147. DeNoyer (1974) has studied this case extensively. A crucial parameter is the radio flux density, which is related to the observed turnover frequency and the distance. Following the argument by DeNoyer, it is possible to explain the observed radio spectrum of $\mathrm{S} 147$, in particular, for the smaller distance of $\leq 1 \mathrm{kpc}$.

(4) If synchrotron losses of electrons occur uniformly over the whole lifetime of the SNR, the turnover would happen at $v_{\mathrm{c}}(\mathrm{MHz})=3.4 \times 10^{9} \mathrm{~B}(10 \mu \mathrm{G})^{-3} t\left(10^{4} \mathrm{yr}\right)^{-2}$. For S147 the turnover frequency $v_{\mathrm{c}}$ is observed to be at $1.5 \mathrm{GHz}$ and its age is about $10^{5} \mathrm{yr}$, so the magnetic fields should be about $0.3 \mathrm{mG}$. Such a high magnetic field is not known to exist in shell type SNRs. DeNoyer (1974) discusses the situation that the acceleration and synchrotron losses occur during the early phase of SNRs. Gull (1973) has shown that in the early phase Rayleigh-Taylor instabilities create a convective zone, which can sustain magnetic fields $B$ of $1000 \mu \mathrm{G}$ within young remnants for approximately 100 years, corresponding to a radius $R_{\mathrm{c}}$ of the remnant of $\sim 1 \mathrm{pc}$ in the low-density region of the Galactic anti-center. Due to the subsequent expansion, the magnetic field decreases with $\left(R / R_{\mathrm{c}}\right)^{-2}$, while the energy decreases with $\left(R / R_{\mathrm{C}}\right)^{-1}$. The turnover frequency decreases with $\left(R / R_{\mathrm{c}}\right)^{-4}$. For $\mathrm{S} 147, R / R_{\mathrm{c}}$ is 21 for a distance of $800 \mathrm{pc}$ corresponding to a turnover frequency of $12 \mathrm{MHz}$ and $154 \mathrm{MHz}$, respectively. However, as DeNoyer (1974) pointed out, at this stage of evolution the magnetic field of the SNR is dominated by the shock compressed interstellar magnetic field, which may be about $10 \mu \mathrm{G}$ in the large-scale emission area. In that case, the turnover frequency decreases with $\left(R / R_{\mathrm{c}}\right)^{-2} \times(10 \mu \mathrm{G} / 1000 \mu \mathrm{G})$ to about $188 \mathrm{MHz}$ and $680 \mathrm{MHz}$, respectively. Given the uncertainty of the magnetic field strength during the synchrotron loss phase, the interstellar magnetic field in the area of S147, as well as the amount of shock compression (with or without cooling effects), this model cannot be ruled out as a possible explanation of the observed spectral bend.

We conclude, in principle, both the compressed Galactic magnetic field scenario and early high synchrotron losses with subsequent adiabatic expansion could explain the observed turnover. The observed difference of $\Delta \alpha \sim 1$ between the highfrequency and the low-frequency spectral indices is larger than the value given by Tang (1984), but smaller than that from synchrotron losses with no further injection of relativistic electrons after the first 100 years (see Fig. 2.12 in Pacholczyk 1977).

\section{The polarization toward S147}

$\lambda 6 \mathrm{~cm}$ and $\lambda 11 \mathrm{~cm}$ polarization maps are shown in the lower panels of Figs. 1 and 2. The polarization features seen in the two polarization maps are complex, but very similar at both wavelengths. They have no counterpart in the total intensity map except for some sections of the S147 shell. Obviously, the interstellar diffuse emission component is responsible for most of the observed polarization features. The similarity of polarized structures at both wavelengths suggests that Faraday rotation along the line of sight is very small.

We extracted the polarization intensity map of the same region (Fig. 11) from the $\lambda 21 \mathrm{~cm}$ "Effelsberg Medium Galactic Latitude Survey" (EMLS) (Reich et al. 2004). This map also includes the large-scale polarized emission component at an absolute zero level from the polarization survey made with the DRAO 26-m telescope (Wolleben et al. 2006). The polarized emission along the bright shell of S147 is very low. The shell of S147 might contribute polarized emission, which adds to the Galactic emission forming minimum polarization. As an alternative S147 might depolarize the emission from larger distances.

Large-scale emission component is missing in the polarization data at $\lambda 6 \mathrm{~cm}$ and $\lambda 11 \mathrm{~cm}$, which is known to severely limit any interpretation of polarization structures caused by Faraday rotation effects in the interstellar medium (Reich 2006). Following Sun et al. (2007) we used the WMAP polarization data at $22.8 \mathrm{GHz}$ to recover the missing large-scale structures at $\lambda 11 \mathrm{~cm}$ and $\lambda 6 \mathrm{~cm}$.

For this purpose, we convolved the WMAP $U$ and $Q$ maps to $49^{\prime}$ and scaled to $\lambda 11 \mathrm{~cm}$ and $\lambda 6 \mathrm{~cm}$ with a temperature spectral index of $\beta=-2.8$, equal to the temperature spectral index toward the Galactic anti-center (Reich \& Reich 1988). We obtained a spectral index of the polarized emission toward S147 of $\beta=-3.0 \pm 0.4$ from a $T-T$ plot between the $\lambda 21 \mathrm{~cm}$ map and the WMAP $22.8 \mathrm{GHz}$ map, which are both on an absolute scale. Although the uncertainty is large, the spectrum of the polarized emission is consistent with that for total intensity, implying that the polarized emission at longer wavelengths is not much reduced by depolarization and that a low Faraday rotation occurs 


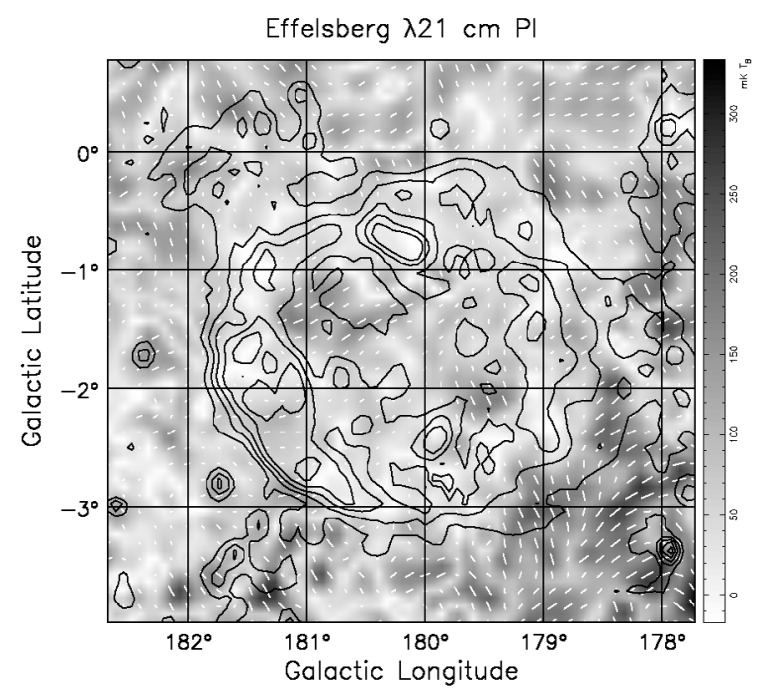

Fig. 11. $\lambda 21 \mathrm{~cm}$ polarization intensity map of S147 from the Effelsberg $100-\mathrm{m}$ telescope at an angular resolution of 9'.4 (grey scale). The rmsnoise is $8 \mathrm{mK} T_{\mathrm{B}}$. Contours show total intensities (the strong point-like sources were subtracted) starting from $150 \mathrm{mK} T_{\mathrm{B}}$ and increasing in steps of $100 \mathrm{mK} T_{\mathrm{B}}$. The bars show the orientation of the magnetic field $\left(\mathrm{E}+90^{\circ}\right)$. The length of the bars is proportional to the polarized intensity.

in the direction of the Galactic anti-center. This justifies the usage of the better known temperature spectral index $\beta=-2.8$.

The difference of the scaled WMAP data and the $\lambda 11 \mathrm{~cm}$ and $\lambda 6 \mathrm{~cm}$ maps, which were convolved to the same beam, are then added to the original $U$ and $Q$ maps at $\lambda 6 \mathrm{~cm}$ and $\lambda 11 \mathrm{~cm}$ wavelength. This procedure needs, however, to be modified in case of significant Faraday rotation along the line of sight in particular for the $\lambda 11 \mathrm{~cm}$ map. We have also used the $\lambda 21 \mathrm{~cm}$ (Fig. 11) convolved to a $49^{\prime}$ beam and the WMAP $22.8 \mathrm{GHz}$ map to obtain the average Faraday rotation over the entire area. We obtain a value of $\sim+10 \mathrm{rad} \mathrm{m}^{-2}$. A low Faraday rotation is also supported by the pulsar PSR J0538+2817, associated with S147, which has a Faraday rotation of $-7 \pm 12 \mathrm{rad} \mathrm{m}^{-2}$ (Mitra et al. 2003). We conclude that the correction of the polarization angle at $\lambda 11 \mathrm{~cm}$ is smaller than a few degrees. In the following, we assume that no modification of the straightforward procedure is necessary.

As expected for a magnetic field orientation along the Galactic plane a large offset is found for Stokes $Q$ (average $\sim 4 \mathrm{mK} T_{\mathrm{B}}$ at $\lambda 6 \mathrm{~cm}$ ), while the offset for Stokes $U$ is small (average $\sim-0.2 \mathrm{mK} T_{\mathrm{B}}$ at $\lambda 6 \mathrm{~cm}$ ). The polarized intensity maps at $\lambda 11 \mathrm{~cm}$ and $\lambda 6 \mathrm{~cm}$, after restoration for an absolute zero level, are shown in Figs. 12 and 13. The strongest polarized emission is located outside the boundary of S147 in both maps. The distribution of polarized intensity in the $\lambda 6 \mathrm{~cm}$ map does not allow the identification of S147 in general. Only a few filaments, e.g., $l, b=181^{\circ} .6,-1^{\circ} .0$ and $l, b=181^{\circ} .2,-2.0$, show an association between total intensity filaments and depressions in polarized intensity. In the $\lambda 11 \mathrm{~cm}$ map, several filaments are clearly visible as depressions in polarized intensity. At both frequencies, the appearance of S147 against the large-scale polarized emission component of the Galactic background is similar to its appearance at $\lambda 21 \mathrm{~cm}$ (see Fig. 11), but the "depolarization" effect is less serious at higher frequencies.

The interpretation of the restored polarization data requires modeling of the diffuse Galactic polarized emission component including Faraday rotation effects (Reich 2006). However, the strong eastern filamentary structure is a region where polarized emission from the SNR adds to the Galactic emission. It

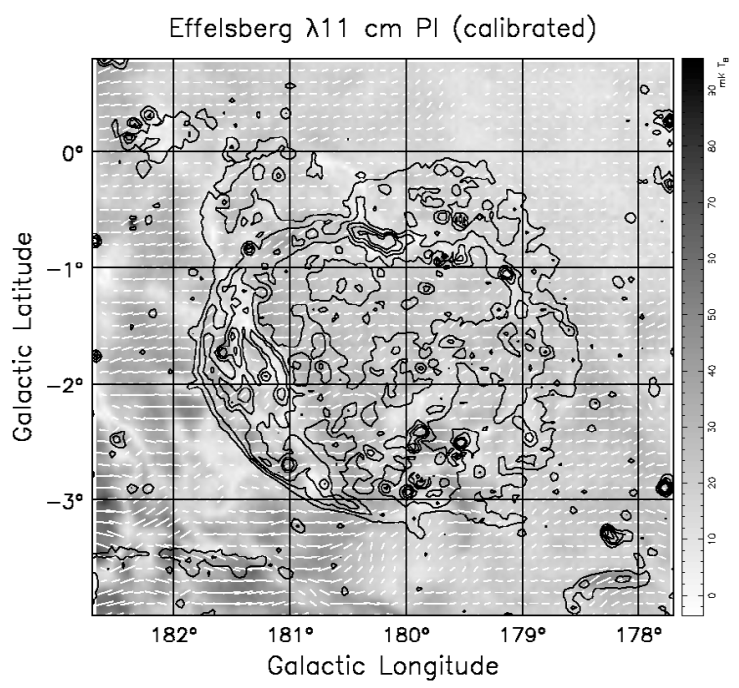

Fig. 12. Polarized $\lambda 11 \mathrm{~cm}$ emission in the area of S147 after restoration for an absolute zero-level using WMAP $22.8 \mathrm{GHz}$ data (grey scale). The angular resolution is 4.4. Contours show total intensities from the Effelsberg $\lambda 11 \mathrm{~cm}$ map (with strong point-like sources subtracted). The bars show the orientation of the magnetic field $\left(\mathrm{E}+90^{\circ}\right)$ in case of ignorable Faraday rotation. The length of the bars is proportional to the polarized intensity.

produces a depression in the polarized intensity map because the magnetic field along the filaments is oriented almost perpendicular to the general Galactic magnetic field direction. The strong polarized filament structure is visible in the original polarization map at a relative zero level (Figs. 1 and 2).

\subsection{Rotation measures of the eastern filaments}

For the calculation of rotation measures (RMs) of S147 we have to remove the diffuse Galactic polarized emission component. We noticed that the polarized emission from the eastern filament is strong and outstanding against the background in the original polarization map. We calculated the RM map from the polarization angles of the original $\lambda 11 \mathrm{~cm}$ and $\lambda 6 \mathrm{~cm}$ polarization maps after both maps have been convolved to a common angular resolution of $10^{\prime}$. Figure 14 shows the RM distribution $(n=0)$ for the eastern filamentary region. We have excluded pixels with a polarized intensity below $1.40 \mathrm{mK} T_{\mathrm{B}}$ at $\lambda 11 \mathrm{~cm}$ or $0.75 \mathrm{mK} T_{\mathrm{B}}$ at $\lambda 6 \mathrm{~cm}$.

In principle, we require polarization maps at a minimum of three frequencies to determine the unambiguous RMs. However, we cannot use the $\lambda 21 \mathrm{~cm}$ map for this purpose because the large-scale diffuse structure dominates and the filaments are not clearly visible. RMs calculated at $\lambda 6 \mathrm{~cm}$ and $\lambda 11 \mathrm{~cm}$ have an ambiguity of $\pm n \times 362 \mathrm{rad} \mathrm{m}^{-2}(n=0, \pm 1, \pm 2, \ldots)$. Any value of $n$ other than zero seems unlikely toward the Galactic anticenter because the direction of the local magnetic field is almost perpendicular to the line of sight and thus small RM values are expected.

The average RM in the east filamentary region is found to be $-70 \pm 8 \mathrm{rad} \mathrm{m}^{-2}$, which is almost identical to the $R M=$ $-75 \pm 4 \mathrm{rad} \mathrm{m}^{-2}$ of an extragalactic source, $0539+266 \mathrm{~B}$, shining through this region (Kim 1988). Outside the filamentary shell, the RM excess caused by S147 is likely rather small, as indicated by the smooth distribution of polarized intensity and polarization angles in Fig. 12. As was mentioned previously the average Faraday rotation over the entire area is low. Therefore, 


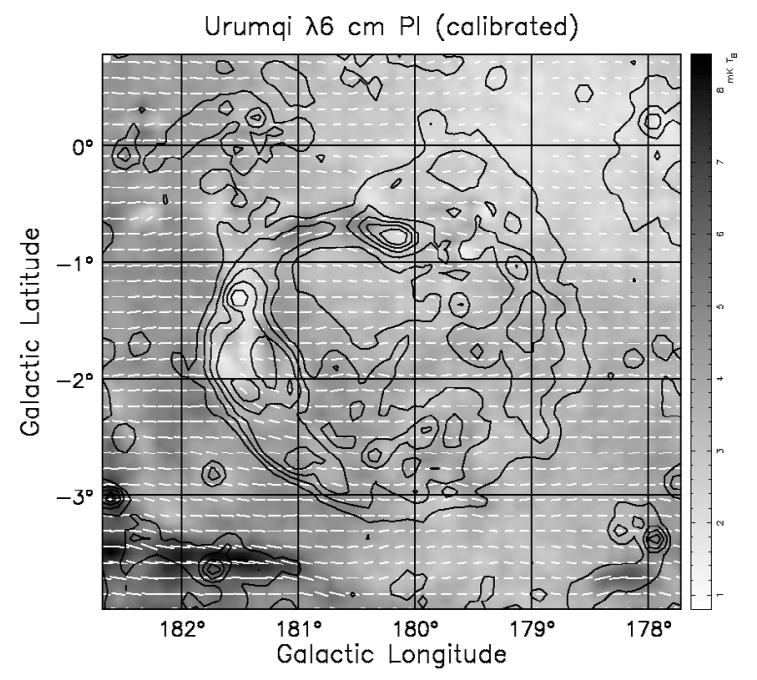

Fig. 13. Same at Fig. 12 but for Urumqi $\lambda 6 \mathrm{~cm}$ map. The angular resolution is 9.5 .

the intrinsic magnetic field vectors of the eastern filaments are very close to the magnetic field direction shown in Fig. 1.

\subsection{Magnetic fields of the eastern filament}

We estimate the magnetic field strength in the eastern filamentary region by assuming energy equipartition between the magnetic field and electrons and protons (Fürst \& Reich 2004):

$B_{\min } \approx 10 \cdot \Phi \cdot R(\mathrm{deg})^{-6 / 7} \cdot d(\mathrm{kpc})^{-2 / 7} \cdot S_{1 \mathrm{GHz}}(\mathrm{Jy})^{2 / 7}$.

We integrated the filamentary region and obtained a flux density at $\lambda 6 \mathrm{~cm}$ of $5.1 \mathrm{Jy}$. The flux density at $1 \mathrm{GHz}$ is extrapolated using the spectral index of $\alpha=-0.35$. The radius of the radiating region is assumed to be 0.5 and the fraction of the radiation volume $\Phi$ is $\sim 1$. For distances of $0.8 \mathrm{kpc}$, we obtain an estimate for the magnetic field strength of about $36 \mu \mathrm{G}$. Using the $\mathrm{RM}$ value $-70 \mathrm{rad} \mathrm{m}^{-2}$ for the eastern filamentary region, we estimated the line of sight component of the intrinsic magnetic field. Assuming an electron density of $100 \mathrm{~cm}^{-3}$ within the filaments and their thickness to be $1^{\prime \prime} \sim 0.07$ pc (Fesen et al. 1985), we calculate $B_{\|} \approx 35 \times \mathrm{RM} / n_{\mathrm{e}} \sim 24 \mu \mathrm{G}$. Both values are of the same order. Magnetic fields in these filamentary structures are typically $30 \mu \mathrm{G}$.

\section{Summary}

We present new sensitive maps of S147 for the total and polarized intensity at $\lambda 6 \mathrm{~cm}$ and $\lambda 11 \mathrm{~cm}$. The $\lambda 6 \mathrm{~cm}$ maps are the first complete ones obtained so far. Our new measurements confirm the turnover of the integrated spectrum of S147 at a frequency of about $1.5 \mathrm{GHz}$. The measured structures have been decomposed into filamentary structures (smaller than $18^{\prime}$ ) and large-scale diffuse emission component. The $T-T$ plots between $2639 \mathrm{MHz}$ and $4800 \mathrm{MHz}$ for these two emission components prove that the turnover is attributed entirely to the diffuse emission component, which has a spectral index of $\alpha \sim-1.35$. The filamentary emission component has a spectral index of $\alpha \sim-0.35$ and can be traced up to $40.7 \mathrm{GHz}$ on WMAP maps.

We discussed several possible mechanisms to explain the turnover of the diffuse emission component. We can exclude the

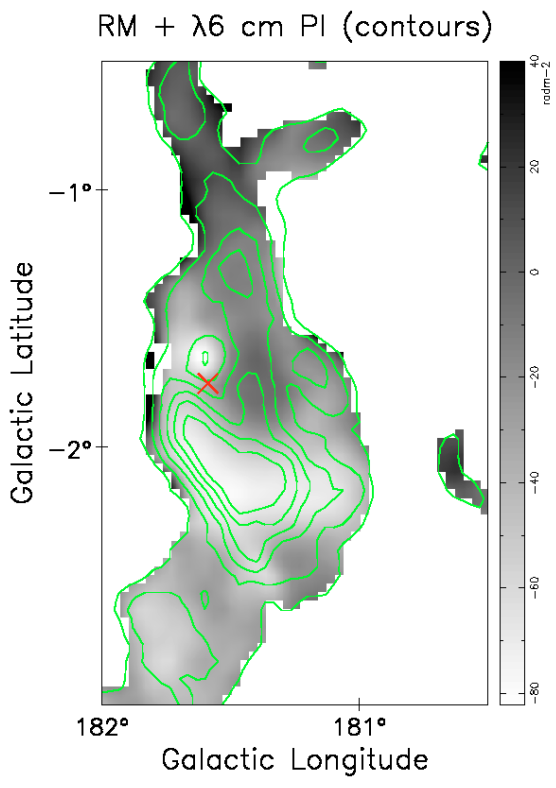

Fig. 14. The RM distribution for the eastern filamentary emission region of S147 derived from the original $\lambda 11 \mathrm{~cm}$ and $\lambda 6 \mathrm{~cm}$ polarization maps. Contours show polarized intensities from the Urumqi $\lambda 6 \mathrm{~cm}$ map starting at $2.5 \mathrm{mK} T_{\mathrm{B}}$ and running in steps of $0.8 \mathrm{mK} T_{\mathrm{B}}$. The position of the extragalactic source $0539+266 \mathrm{~B}$ with a RM of $-75 \pm 4 \mathrm{rad} \mathrm{m}^{-2}$ (Kim 1988) is indicated.

scenarios of two populations of electrons as well as the diffuse shock accelerated of electrons. However, a compressed magnetic field is a possible explanation. The SNR shock wave compresses the local magnetic field and shifts the turnover in the Galactic radio spectrum from about $400 \mathrm{MHz}$ to about $1.5 \mathrm{GHz}$. High synchrotron losses, during the early phase of the SNR, would cause a bend at a rather high frequency, but subsequent expansion of S147 would shift the frequency toward about $1.5 \mathrm{GHz}$ as well.

S147 is a faint source located on the near side of the Perseus arm. Polarized emission from S147 is confused with large-scale Galactic emission, except for the bright filamentary regions. The $\mathrm{RM}$ in the eastern filamentary region is about $-70 \mathrm{rad} \mathrm{m}^{-2}$. The magnetic field in the filamentary region has a strength of typically $\sim 30 \mu \mathrm{G}$.

Acknowledgements. We acknowledge travel support from the MPG and CAS exchange program. The $\lambda 6 \mathrm{~cm}$ observations of S147 were carried out at the NanShan station of Urumqi observatory of NAOC. We thank all the people involved in the installation of the $\lambda 6 \mathrm{~cm}$ receiver and the required observing telescope software, in particular, Mr. Otmar Lochner for the construction of the receiver, and Dr. Peter Müller and Dr. Xiaohui Sun for software installation and development at the telescope. LX would like to thank Dr. Xiaohui Sun for qualified support on all kinds of data reduction related issues. We thank Mr. M. Z. Chen, Mr. J. Ma, and the staff of the Urumqi Observatory for maintenance and assistance during the observations. The $\lambda 11$ data are based on observations with the $100 \mathrm{~m}$ telescope of the Max-Planck-Institut für Radioastronomie at Effelsberg. The research work of LX and JLH are supported by the National Natural Science Foundation of China (10473015, 10521001 and 10773016) and by the Partner group of MPIfR at NAOC. We would like to thank Richard Wielebinski and Patrica Reich for critical reading of the manuscript.

\section{References}

Anderson, S. B., Cadwell, B. J., Jacoby, B. A., et al. 1996, ApJ, 468, 55 Clark, D. H., \& Caswell, J. L. 1976, MNRAS, 174, 267 
DeNoyer, L. K. 1974, AJ, 79, 1263

D'Odorico, S., \& Sabbadin, F. 1977, AAS, 28, 439

Ellison, D., \& Reynolds, S. 1991, ApJ, 382, 242

Emerson, D. T., \& Gräve, R. 1988, A\&A, 190, 353

Fesen, R. A., Blair, W. P., \& Kirshner, R. P. 1985, ApJ, 292, 29

Finkbeiner, D. P. 2003, ApJS, 146, 407

Fürst, E., \& Reich, W. 1986, A\&A, 163, 185

Fürst, E., \& Reich, W. 2004, in The Magnetized Interstellar Medium, ed. B.

Uyanıker, W. Reich, \& R. Wielebinski (Copernicus GmbH), 141

Fürst, E., Reich, W., Beck, R., Hirth, W., \& Angerhofer, P. E. 1982, A\&A, 115, 428

Gull, S. F. 1973, MNRAS, 161, 47

Haslam, C. G. T. 1974, A\&AS, 15, 333

Hinshaw, G., Nolta, M. R., Bennett, C. L., et al. 2007, ApJS, 170, 288

Kim, K.-T. 1988, J. Kor. Astron. Soc., 21, 133

Kirshner, R. P., \& Arnold, C. N. 1979, ApJ, 229, 147

Kovalenko, A. V., Pynzar, A. V., \& Udal'tsov, V. A. 1994, AR, 38, 95

Kundu, M. R., Angerhofer, P. E., Fürst, E., \& Hirth, W. 1980, A\&A, 398, 993

Leahy, D. A., \& Roger, R. S. 1998, ApJ, 505, 784

Milne, D. K. 1979, Austr. J. Phys., 32, 83

Minkowski, R. 1958, Rev. Mod. Phys., 30, 1048

Mitra, D., Wielebinski, R., Kramer, M., \& Jessner, A. 2003, A\&A, 398, 993

Muders, D., Polehampton, E., \& Hatchell, J. 2007, Multi-beam Fits Raw Data Format, APEX Report APEX-MPI-IFD-002, Rev. 1.61

Ng, C. Y., Romani, R. W., Brisken, W. F., Chatterjee, S., \& Kramer, M. 2007, ApJ, 654, 487

Pacholczyk, A. G. 1977, Radio Galaxies (Pergamon Press)
Phillips, A. P. Gondhalekar, P. M. \& Blades, J. C. 1981, MNRAS, 195, 485 Reich, P., \& Reich, W. 1988, A\&AS, 74, 7

Reich, W. 2006, in Cosmic Polarization 2006, ed. R. Fabbri (Research Signpost), 91

Reich, W., Fürst, E., Reich, P., et al. 2004, in The Magnetized Interstellar Medium, ed. B. Uyanıker, W. Reich, \& R. Wielebinski (Katlenburg-Lindau: Copernicus $\mathrm{GMbH}), 57$

Reich, W., Zhang, X., \& Fürst, E. 2003, A\&A, 408, 961

Sallmen, S., \& Welsh, B. Y. 2004, A\&A, 426, 555

Sofue, Y., \& Reich, W. 1979, A\&AS, 38, 251

Sofue, Y., Fürst, E., \& Hirth, W. 1980, PASJ, 32, 1

Sun, X. H., Reich, W., Han, J. L., Reich, P., \& Wielebinski, R. 2006, A\&A, 447, 947

Sun, X. H., Han, J. L., Reich, W., et al. 2007, A\&A, 463, 993

Tang, K. 1984, ApJ, 278, 881

Trushkin, S. A. 2002, http: //cats. sao.ru

Turtle, A. J., Pugh, G. F., Kenderdine, S., \& Pauliny-Toth, I. I. K. 1962, MNRAS, 124, 297

Uyanıker, B., Fürst, E., Reich, W., Reich, P., \& Wielebinki, R. 1999, A\&AS, 138,31

van den Bergh, S. 1960, Z. Astrophys., 51, 15

Wardle, J. F. C., \& Kronberg, P. P. 1974, ApJ, 194, 249

Webster, A. 1974, MNRAS, 155, 355

Wolleben, M., Landecker, T. L., Reich, W., \& Wielebinski, R. 2006, A\&A, 488, 411

Zhang, X., Reich, W., Reich, P., \& Wielebinski, R. 2003, A\&A, 404, 57 